\title{
DISTRIBUIÇÃO DE WEIBULL E AVALIAÇÃO ABNT DE COM TIJOLO DE ARGILA PARA ALVENARIA ESTRUTURAL USADO EM CONSTRUÇÕES DE ATÉ 5 ANDARES*
}

\author{
Neila Azeredo Gondin ${ }^{1}$ \\ Jonas Alexandre ${ }^{1}$ \\ Afonso Rangel Garcez Azevedo 2 \\ Gustavo de Castro Xavier ${ }^{1}$ \\ Sergio Neves Monteiro ${ }^{3}$ \\ Leonardo Gonçalves Pedroti ${ }^{4}$ \\ Fernando Cesar Coelho França ${ }^{1}$
}

Resumo

Este trabalhou lidou com a avaliação de amostras de tijolo de argila para alvenaria estrutural com adição de resíduo cerâmico cujas dimensões nominais são $11,5 \times 39,0 \times 19,0 \mathrm{~cm}$. As amostras foram queimadas nas temperaturas $700^{\circ} \mathrm{C}$ e $800^{\circ} \mathrm{C}$. Elas foram coletadas em uma fábrica de cerâmica vermelha em Campos dos Goytacazes, RJ, Brasil. De um lote, a amostra contendo 20 tijolos de alvenaria já queimada em seu forno a $700^{\circ} \mathrm{C}$ foi coletada, e também a amostra contendo 20 tijolos de alvenaria do mesmo lote secos apenas pelo ar, que foram queimadas em um forno de mufla a uma temperatura de $800^{\circ} \mathrm{C}$. As amostras foram submetidas aos testes recomendados pela ABNT NBR 15270: 2005 para determinar suas propriedades geométricas, físicas e mecânicas. A ABNT NBR 15270: 2005 recomenda formulações específicas para determinar a resistência à compressão característica (fbk) da alvenaria de tijolos de argila que, para ser classificada, deve ser de pelo menos $3 \mathrm{MPa}$. Portanto, também usamos o Método Gráfico de Probabilidade e o Método da Máxima Verossimilhança para estimar os parâmetros de distribuição para comparar os resultados obtidos nos testes realizados.

Palavras-chave:Tijolos de argila, Alvenaria estrutural, Análise de Weibull.

\section{WEIBULL DISTRIBUTION AND BRAZILIAN ABNT STANDARD EVALUATION OF STRUCTURAL CLAY BRICK MASONRY UNITS USED IN UP TO 5 FLOORS CONSTRUCTION}

\section{Abstract}

This work dealt with the evaluation of structural clay brick masonry samples with the addition of ceramic residue which nominal dimensions are $11.5 \times 39.0 \times 19.0 \mathrm{~cm}$. The samples were burned at temperatures of $700^{\circ} \mathrm{C}$ and $800^{\circ} \mathrm{C}$. They were collected in a red-brick plant in Campos dos Goytacazes, RJ, Brazil. From a lot, a sample containing 20 clay bricks masonry already burned in its furnace at $700^{\circ} \mathrm{C}$ was collected, and also a sample containing 20 clay bricks masonry from the same lot only dried by the air, which were then burned in a muffle furnace at a temperature of $800^{\circ} \mathrm{C}$. The samples were subjected to the tests recommended by ABNT NBR 15270:2005 to determine their geometric properties, physical and mechanical. The ABNT NBR 15270:2005 recommends specific formulations to determine the characteristic compressive strength (fbk) from clay brick masonry which, to be classified as structural clay brick masonry should be at least $3 \mathrm{MPa}$. Therefore, we also used the Probability Plot Method and Likelihood Method to estimate distribution parameters in order to compare the results obtained in the tests conducted.

Keywords: Clay Brick, Masonry, Weibull analysis.

Laboratório de Engenharia Civil (LECIV), Universidade Estadual do Norte Fluminense (UENF), Av. Alberto Lamego, 2000, Campos dos Goytacazes, Rio De Janeiro, Brazil. 
2 Laboratório de Engenharia Civil (LECIV), Universidade Estadual do Norte Fluminense (UENF), Av. Alberto Lamego, 2000, Campos dos Goytacazes, Rio De Janeiro, Brazil. afonso.garcez91@gmail.com.

3 Seção de Engenharia Mecânica e de Materiais, Instituto Militar de Engenharia (IME), Praça General Tibúrcio, 80, URCA, Rio de Janeiro, Brazil.

4 Universidade Federal de Viçosa (UFV), Avenida P. H. Rolfs s/n, Viçosa, Minas Gerais, Brazil. 


\section{INTRODUÇÃO}

O Modelo de Frequência de Weibull se aplica a diversos problemas de Engenharia, destacando-se a determinação da vida útil ou da resistência de peças. Sua formulação permite uma plasticidade que emula a maior parte dos modelos de frequência. Esse modelo é empregado na caracterização de materiais frágeis tais como os cerâmicos [9].

Este trabalho se propõe avaliar amostras de blocos cerâmicos estruturais fabricados com adição de resíduos cerâmicos com dimensões nominais de $11,5 \times 39,0 \times 19,0 \mathrm{~cm}$, queimados a temperaturas de $700^{\circ} \mathrm{C}$ e $800^{\circ} \mathrm{C}$ a partir das recomendações da norma técnica [8] e a partir do Modelo de Frequência de Weibull.

As amostras de blocos cerâmicos estruturais foram submetidas aos ensaios recomendados para determinação de suas propriedades geométricas, físicas e mecânicas $[2,3,4,5,6,7,8]$. A ABNT NBR 15270:2005 recomenda formulações específica para a determinação da resistência característica à compressão $\left(f_{b k}\right)$ dos blocos cerâmicos, que para serem classificados como estrutural devem ter no mínimo $3 \mathrm{MPa}$. Nesse sentido, utilizou-se o Método Gráfico de Probabilidade e o Método da Máxima Verossimilhança como estimador dos parâmetros que compõe a distribuição de Weibull para se comparar os resultados obtidos nas duas abordagens.

\subsection{Distribuição de frequência de Weibull}

A função de densidade de probabilidade de Weibull é definida como [9]:

$$
f(x)=\frac{\beta(x-\delta)^{\beta-1}}{\theta^{\beta}} e^{\left[-\left(\frac{x-\delta}{\theta}\right)^{\beta}\right]}
$$

Onde: $\beta$ é o parâmetro (fator) de forma, ou Módulo de Weibull; $\theta$ é o parâmetro de escala; $\delta$ é o parâmetro de localização.

O parâmetro de localização nesta análise foi estipulado como zero, uma vez que $\delta \neq 0$ altera drasticamente os valores de $\beta$ e $\theta$. O parâmetro de localização refere-se a uma grandeza inicial, sendo o recomendado para a atual análise que seja adotado $\delta=0$.

A média de distribuição de Weibull é definida como [9]:

$$
E(x)=\delta+\theta \Gamma\left(1+\frac{1}{\beta}\right)
$$

Onde: $\Gamma$ é a Função Gama, tabulada em textos estatísticos.

A variância da distribuição de Weibull é definida como [9]:

$$
\mathrm{V}(\mathrm{x})=\theta^{2}\left[\Gamma\left(1+\frac{2}{\beta}\right)+\Gamma^{2}\left(1+\frac{1}{\beta}\right)\right]
$$

A função de Confiabilidade descreve a probabilidade de sobrevivência em função do tempo, ou de fratura em função de tensão. Essa função é definida como [9]:

$$
R(x)=e^{\left[-\left(\frac{x-\delta}{\theta}\right)^{\beta}\right]}
$$

A função de Risco representa a taxa de falha instantânea e pode ser usada para caracterizar falhas ocorridas. Essa função é definida como [9]:

$$
H(x)=\frac{\beta(x-\delta)^{\beta-1}}{\theta^{\beta}}
$$


As estimativas de parâmetros de Weibull podem ser feitas por alguns métodos, como: Gráfico de Probabilidade, Gráfico de Risco, Máxima Verossimilhança, Estimadores de Momento e Estimadores Lineares. Neste trabalho foi utilizado o Método Gráfico de Probabilidade e o Método da Máxima Verossimilhança como estimador dos parâmetros que compõe a distribuição de Weibull.

\subsection{Distribuição de Rank}

Dada uma sequência ordenada de dados, $\mathrm{x}_{1}, \mathrm{x}_{2}, \ldots, \mathrm{x}_{\mathrm{n}}$ cujo número total de amostras é $\mathrm{n}$ e tem uma função de distribuição cumulativa $F(x)$, onde $x$ é um valor contínuo, deseja-se determinar os valores dos estimadores $F\left(x_{1}\right), F\left(x_{2}\right), \ldots, F\left(x_{n}\right)$. Para se estimar o valor de $F\left(x_{n}\right)$, neste trabalho, adotou-se o Rank Mediano. O Rank Mediano é definido pela expressão[9]:

$$
\text { Rank Mediano } \rightarrow\left(x_{j}\right)=\frac{j-0,3}{n+0,4}
$$

Onde: j é a ordem da amostra; $\mathrm{n}$ é o número total de amostras em um lote.

\subsection{Intervalo de Confiança}

O Teorema do Limite Central prevê que quanto maior for o número de amostras estudado, mais a distribuição desse número tende a ser uma Normal. Para esse estudo foram utilizados 13 amostras para cada temperatura de queima, uma vez que a ABNT NBR 15270:2005 recomenda esse número para ensaio de resistência à compressão. No entanto, um número pequeno de amostras pode ser utilizado no Modelo de Weibull com estimativa de limites de confiança quase exatas[14].

\subsubsection{Intervalos de Confiança para o Parâmetro de Forma $(\beta)$}

Um dos métodos para a determinação do intervalo de confiança de $\beta$ é o da Máxima Verossimilhança, que é o empregado neste trabalho. $O$ valor de $\beta$ é estimado fazendo-se com que os dois lados da equação abaixo sejam iguais [10];

$$
\sum_{i=1}^{r} \frac{\ln \left(x_{i}-\delta\right)}{r}=\frac{\sum_{i=1}^{r}\left(x_{i}-\delta\right)^{\beta} \ln \left(x_{i}-\delta\right)}{\sum_{i=1}^{r}\left(x_{i}-\delta\right)^{\beta}}-\frac{1}{\beta}
$$

Os limites de confiança são da seguinte forma:

$$
\beta_{\text {inf } \text { erior }}=\frac{\beta}{\exp \left(\frac{K \sqrt{F_{(2,2)}^{-1}}}{\beta}\right)}
$$

Onde: K é o [100(1 - $\alpha / 2)]$-ésimo percentil normal para 100(1- $\alpha)$ limite percentual. Para um limite de $95 \%$, como empregado neste estudo, K é igual ao 97.5-ésimo percentil normal, que equivale a 1.96. Para o limite superior [10]:

$$
\beta_{\text {sup erior }}=\beta \exp \left(\frac{\mathrm{K} \sqrt{\mathrm{F}_{(2,2)}^{-1}}}{\beta}\right)
$$


F é a matriz composta dos seguintes elementos:

Onde:

$$
F=\left(\begin{array}{cc}
-\frac{\partial L_{T}^{2}}{\partial \theta^{2}} & -\frac{\partial L_{T}^{2}}{\partial \beta \partial \theta} \\
-\frac{\partial L_{T}^{2}}{\partial \beta \partial \theta} & -\frac{\partial L_{T}^{2}}{\partial \beta^{2}}
\end{array}\right)
$$

$$
\begin{array}{r}
\frac{\partial \mathrm{L}_{\mathrm{T}}^{2}}{\partial \theta^{2}}=\frac{\beta}{\theta^{2}}-\left(\frac{\mathrm{x}_{\mathrm{i}}}{\theta}\right)^{\beta}\left(\frac{\beta}{\theta^{2}}\right)(\beta+1) \\
\frac{\partial \mathrm{L}_{\mathrm{T}}^{2}}{\partial \beta^{2}}=\frac{-1}{\beta^{2}}-\left(\frac{\mathrm{x}_{\mathrm{i}}}{\theta}\right)^{\beta}\left[\ln \left(\frac{\mathrm{x}_{\mathrm{i}}}{\theta}\right)\right]^{2} \\
\frac{\partial \mathrm{L}_{\mathrm{T}}^{2}}{\partial \beta \partial \theta}=\frac{-1}{\theta}+\left(\frac{\mathrm{x}_{\mathrm{i}}}{\theta}\right)^{\beta}\left(\frac{1}{\theta}\right)\left[\beta \ln \left(\frac{\mathrm{x}_{\mathrm{i}}}{\theta}\right)+1\right]
\end{array}
$$

\subsubsection{Intervalos de Confiança para o Parâmetro de Escala ( $(\theta)$}

O Método da Máxima Verossimilhança estima o valor de $\theta$ a partir da seguinte expressão[10]:

$$
\theta=\left(\sum_{i=1}^{r} \frac{\left(x_{i}-\delta\right)^{\beta}}{r}\right)^{\frac{1}{\beta}}
$$

Da mesma forma que no parâmetro anterior, seus limites são:

$$
\begin{aligned}
& \theta_{\text {inf erior }}=\frac{\theta}{\exp \left(\frac{\mathrm{K} \sqrt{F_{(1,1)}^{-1}}}{\theta}\right)} \\
& \theta_{\text {superior }}=\theta \exp \left(\frac{K \sqrt{F_{(1,1)}^{-1}}}{\theta}\right)
\end{aligned}
$$

\subsubsection{Intervalos de Confiança para o Parâmetro de Localização (ర)}

Este estudo adotará o Parâmetro de Localização (ס) com o valor zero, segundo recomendações de Dodson [14]. Será empregado um software acadêmico [14] que implementa o Método da Máxima Verossimilhança, assim como o Método Gráfico de Probabilidades, dentre outros.

\subsection{Resistência à compressão do bloco estrutural cerâmico}

O município de Campos dos Goytacazes ocupa uma área de 4.026,712 $\mathrm{km}^{2}$ do território Norte do Estado do Rio de Janeiro, Brasil, com uma população de 463.731 habitantes [11]. Uma das atividades econômicas da região é a produção de cerâmica vermelha praticada por 98 indústrias associadas ao Sindicato dos Ceramistas - segundo o próprio -, e outras tantas não associadas. Essa produção gira em torno de artefatos, como: lajotas para lajes, tijolos, telhas, revestimentos cerâmicos, blocos estruturais cerâmicos, entre outros. Os processos 
de queima, em grande parte, são pautados na queima de biomassa, como, por exemplo: eucalipto, cavaco (lenha picada), pinho, sabiá, cascas, bagaço e palhas de cana, capim elefante, dentre outros. Nesse sentido, este estudo teve por objetivo ensaiar blocos estruturais cerâmicos com dimensões de fabricação de $11,5 \times 19 \times 39 \mathrm{~cm}$, queimados a temperaturas de $700^{\circ} \mathrm{C}$ e $800^{\circ} \mathrm{C}$ para comparação dos resultados de resistência à compressão [8].

\section{MATERIAIS E MÉTODOS}

Os blocos cerâmicos estruturais para o estudo de resistência à compressão foram fornecidos por uma das indústrias do município de Campos dos Goytacazes. A fabricação de tais blocos se dá mediante extrusão utilizando-se boquilha apropriada, sendo considerados blocos cerâmicos estruturais aqueles produzidos para serem assentados com furos na vertical [8], conforme Figura 1
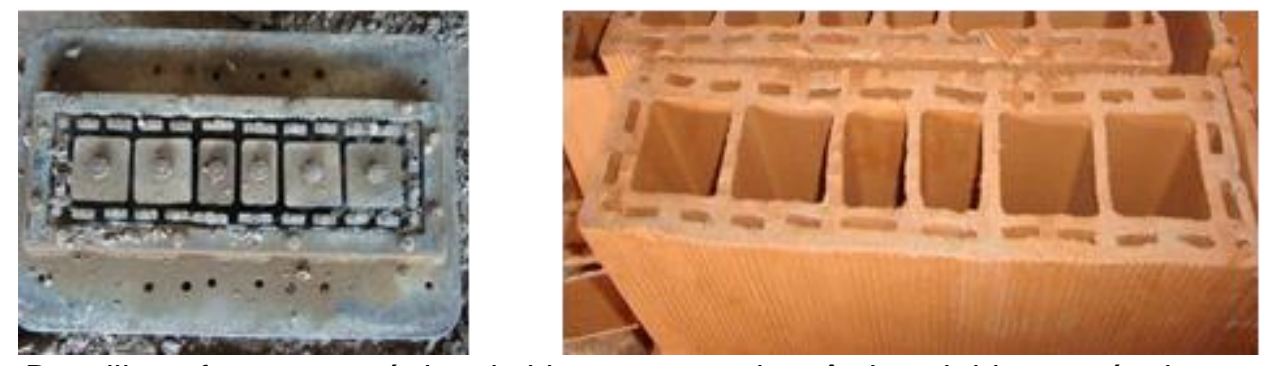

Figura 1. Boquilha e forma geométrica do bloco estrutural cerâmico obtido através de extrusão

Foram coletados 20 blocos de um lote da indústria citada já queimados em seus fornos a $700^{\circ} \mathrm{C}$. Outros 20 blocos, secos ao ar, foram também coletados para o estudo em laboratório, sendo queimados à $800^{\circ} \mathrm{C}$ em um forno tipo Mufla.

Os blocos foram ensaiados segundo a norma [8], que tem como recomendação o ensaio de 13 blocos em um lote, sendo considerado lote o número de blocos de 1.000 a 100.000 . A coleta de 20 blocos deu-se por uma questão de segurança em relação aos possíveis danos no transporte ou manuseio dos elementos.

\section{RESULTADOS E DISCUSSÃO}

Em relação à matéria-prima utilizada para a confecção dos blocos, foi colhida uma amostra na esteira que a leva para a extrusora, de onde os blocos tomam sua forma, com a finalidade de se proceder à sua caracterização física (Granulometria, Limites de Atterberg, Massa Específica Real $[2,3,4,5,6,7]$. Essa matéria-prima é uma mistura de argila com 0 resíduo cerâmico da própria indústria, que é reaproveitado.

\subsection{Ensaio da matéria-prima constitutiva do bloco estrutural cerâmico}

A matéria-prima constitui-se de $51,80 \%$ de fração argila, $35,50 \%$ de fração silte e $12,70 \%$ de fração areia, sendo classificada como $\mathrm{CH}$ (USCS). Os Limites de Atterberg são: Limite de Liquidez de 59,8\%; Limite de Plasticidade de 32,9\%; Índice de Plasticidade de 27,0\%. A densidade real dos grãos é de $2,69 \mathrm{~g} / \mathrm{cm}^{3}$.

Os valores encontrados para as características físicas revelam-se semelhantes aos verificados por outros pesquisadores $[1,12,15,16]$, evidenciando a tipologia dominante para a argila da região utilizada como matéria-prima para confecção, pela indústria ceramista local, dos mais variados artefatos. 


\subsection{Ensaios das amostras de blocos estruturais}

Por definição [8], as amostras constituem-se em um conjunto de blocos retirados aleatoriamente de um lote para determinação de suas propriedades geométricas, físicas ou mecânicas. No caso em questão, por segurança, foram recolhidos mais blocos que o mínimo necessário para os ensaios.

\subsubsection{Ensaio dimensional}

O ensaio dimensional é uma importante etapa do estudo, uma vez que a característica geométrica dos blocos deve atender aos critérios normativos para que tenham desempenho adequado. Os blocos estruturais devem possuir a forma de um prisma reto [8], as áreas brutas $\left(A_{b}\right)$ - que correspondem as áreas das seções de assentamento delimitadas pelas arestas dos blocos, sem desconto das áreas dos furos - devem ser determinadas, uma vez que esses dados são necessários para o cálculo das resistências à compressão.

Nos blocos cerâmicos estruturais [8] de paredes vazadas, a espessura mínima dos septos deve ser de $7 \mathrm{~mm}$ e das paredes externas deve ser no mínimo de $8 \mathrm{~mm}$, conforme mostrado esquematicamente na Figura 2, cuja tolerância relacionada à média das dimensões efetivas deve ser de $\pm 3 \mathrm{~mm}$.

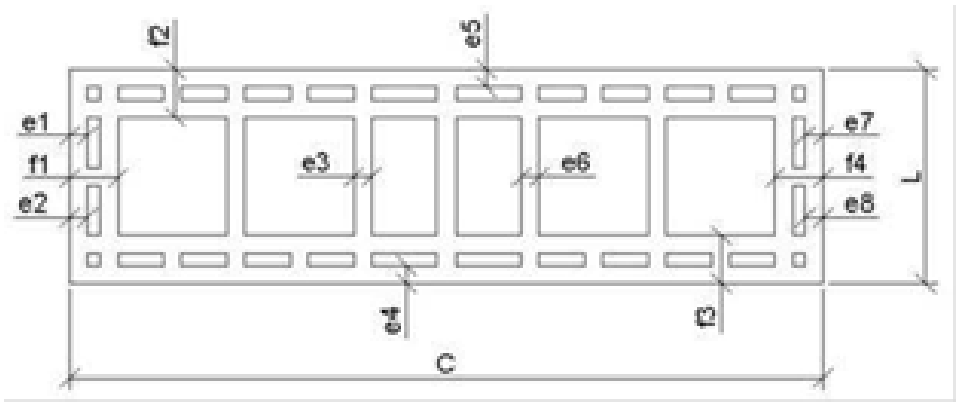

Figura 2. Planta do bloco estrutural de paredes vazadas para levantamento dos dados dimensionais. Fonte: MILLER (2008).

\subsubsection{Ensaio de resistência à compressão}

Na Tabela 1 encontram-se as médias das dimensões efetivas aferidas, as áreas brutas $\left(A_{b}\right)$, as cargas últimas e as resistências à compressão de blocos queimados a $700^{\circ} \mathrm{C}$ e a $800^{\circ} \mathrm{C}$.

Tabela 1. Dimensões, áreas brutas, cargas últimas, tensões últimas.

\begin{tabular}{|c|c|c|c|c|c|c|}
\hline \multirow{2}{*}{$\begin{array}{c}\text { Blocos } \\
700^{\circ} \mathrm{C} \\
\text { (13unidades) }\end{array}$} & \multicolumn{3}{|c|}{ Dimensões (mm) } & \multirow{2}{*}{$\begin{array}{c}\text { Área Bruta } \\
\left(\mathrm{m}^{2}\right)\end{array}$} & \multirow{2}{*}{$\begin{array}{l}\text { Carga } \\
\text { Última } \\
(\mathrm{kN})\end{array}$} & \multirow{2}{*}{$\begin{array}{l}\text { Tensão } \\
\text { Última } \\
\text { (MPa) }\end{array}$} \\
\hline & Largura $(\mathrm{L})$ & Altura $(\mathrm{H})$ & Comprimento (C) & & & \\
\hline Médias & 114,8 & 183,8 & 394,8 & 0,05 & 135,7 & 3,0 \\
\hline Desv. Padrão & 1,48 & 1,48 & 1,48 & 0,000724 & 38,90 & 0,89 \\
\hline Coef.Variação & 1,29 & 0,81 & 0,37 & 1,60 & 28,67 & 29,63 \\
\hline \multirow{2}{*}{$\begin{array}{c}\text { Blocos } \\
800^{\circ} \mathrm{C} \\
\text { (13unidades) }\end{array}$} & \multicolumn{3}{|c|}{ Dimensões (mm) } & \multirow{2}{*}{$\begin{array}{c}\text { Área Bruta } \\
\left(\mathrm{m}^{2}\right)\end{array}$} & \multirow{2}{*}{$\begin{array}{l}\text { Carga } \\
\text { Última } \\
(\mathrm{kN})\end{array}$} & \multirow{2}{*}{$\begin{array}{l}\text { Tensão } \\
\text { Última } \\
(\mathrm{MPa})\end{array}$} \\
\hline & Largura (L) & Altura $(\mathrm{H})$ & Comprimento (C) & & & \\
\hline Médias & 117,2 & 183,8 & 397,9 & 0,05 & 183,42 & 4,0 \\
\hline Desv. Padrão & 1,36 & 2,23 & 2,56 & 0,000782 & 32,35 & 0,69 \\
\hline Coef.Variação & 1,16 & 1,21 & 0,64 & 1,68 & 17,64 & 17,65 \\
\hline
\end{tabular}

A norma [8], no entanto, não adota a média das resistências, mas sim a resistência característica à compressão ( $f_{b k}$ ), calculada conforme sua prescrição, onde é estabelecido como valor mínimo para resistência característica à compressão $\left(f_{b k}\right)$ o valor de $3,0 \mathrm{MPa}$ para que um bloco seja classificado como estrutural, sendo a resistência característica à 
compressão $\left(f_{b k}\right)$ calculada utilizando-se as expressões que se encontram no item 5.5 , página 7, da ABNT NBR 15270-2:2005.

Seguindo as recomendações normativas [8], os valores encontrados para a resistência característica, nesse trabalho, para a temperatura de queima de $700^{\circ} \mathrm{C}$ foi de $\mathrm{f}_{\mathrm{bk}}=1,64 \mathrm{MPa}$; e para a temperatura de queima de $800^{\circ} \mathrm{C}$ foi de $\mathrm{f}_{\mathrm{bk}}=3,03 \mathrm{MPa}$.

\subsection{Análises por meio do Método Gráfico de Probabilidade e Máxima Verossimilhança}

As amostras de blocos queimados a $700^{\circ} \mathrm{C}$ e a $800^{\circ} \mathrm{C}$ foram submetidas à análise pelo Método Gráfico de Probabilidade e pelo Método da Máxima Verossimilhança para determinação dos parâmetros da distribuição estatística de Weibull.

Nas Tabelas 2 e 3 encontram-se os parâmetros de Weibull para os bloco queimados a $700^{\circ} \mathrm{C}$ e $800^{\circ} \mathrm{C}$ respectivamente.

Tabela 2. Parâmetros obtidos pelo Método Gráfico de Probabilidade e pela Máxima Verossimilhança adotando-se $\delta=0$ ao nível de confiança de $95 \%$ para os blocos queimados a $700^{\circ} \mathrm{C}$.

\begin{tabular}{l|c|c|c|c} 
Blocos queimados a 700ㄷ & $\begin{array}{c}\text { Gráfico de } \\
\text { Probabilidade }\end{array}$ & $\begin{array}{c}\text { Máxima } \\
\text { Verossimilhança }\end{array}$ & $\begin{array}{c}\text { Limite } \\
\text { Inferior }\end{array}$ & $\begin{array}{c}\text { Limite } \\
\text { Superior }\end{array}$ \\
\hline Módulo de Weibull $(\beta)$ & 4,098 & 4,196 & 2,970 & 5,928 \\
\hline $\begin{array}{l}\text { Resistência à compressão } \\
\text { característica }(\theta)\end{array}$ & $3,2 \mathrm{MPa}$ & - & $2,83 \mathrm{MPa}$ & $3,56 \mathrm{MPa}$ \\
\hline Média & $2,89 \mathrm{MPa}$ & $2,89 \mathrm{MPa}$ & - & - \\
\hline Desvio Padrão & $0,7932 \mathrm{MPa}$ & $0,7755 \mathrm{MPa}$ & - & - \\
\hline $\mathrm{R}^{2}$ & 0,9019 & - & - & - \\
\hline
\end{tabular}

Tabela 3. Parâmetros obtidos pelo Gráfico de Probabilidade e pela Máxima Verossimilhança adotando-se $\delta=0$ ao nível de confiança de $95 \%$ para os blocos queimados a $800^{\circ} \mathrm{C}$.

\begin{tabular}{l|c|c|c|c}
\hline Blocos queimados a $800^{\circ} \mathrm{C}$ & $\begin{array}{c}\text { Gráfico de } \\
\text { Probabilidade }\end{array}$ & $\begin{array}{c}\text { Máxima } \\
\text { Verossimilhança }\end{array}$ & $\begin{array}{c}\text { Limite } \\
\text { Inferior }\end{array}$ & $\begin{array}{c}\text { Limite } \\
\text { Superior }\end{array}$ \\
\hline Módulo de Weibull $(\beta)$ & 6,233 & 6,377 & 4,508 & 9,021 \\
\hline $\begin{array}{l}\text { Resistência à compressão } \\
\text { característica }(\theta)\end{array}$ & $4,22 \mathrm{MPa}$ & - & $3,91 \mathrm{MPa}$ & $4,55 \mathrm{MPa}$ \\
\hline Média & $3,93 \mathrm{MPa}$ & $3,93 \mathrm{MPa}$ & - & - \\
\hline Desvio Padrão & $0,7345 \mathrm{MPa}$ & $0,7194 \mathrm{MPa}$ & - & - \\
\hline $\mathrm{R}^{2}$ & 0,9368 & - & - & - \\
\hline
\end{tabular}

\section{CONCLUSÕES}

- Os blocos queimados a $700^{\circ} \mathrm{C}$ na indústria, com resistência característica $f_{b k}=1,64$ MPa não atingiram a mínima de 3,0 MPa exigida [8], não podendo, então, serem classificados como estruturais.

- Os blocos queimados a $800^{\circ} \mathrm{C}$, com resistência característica $\mathrm{f}_{\mathrm{bk}}=3,03 \mathrm{MPa}$ atingiram a resistência mínima recomendada por norma [8], estando, assim, em conformidade. Ressalta-se, que em 2003 foi elaborado um documento [13] com o objetivo de estabelecer os critérios e exigências para a construção da estrutura de edifícios habitacionais, quando esta for constituída, essencialmente, de paredes resistentes de alvenaria nos empreendimentos objeto de financiamento, ou contratação pela Caixa Econômica Federal. O documento elaborado refere-se a edifícios residenciais de 3 a 5 pavimentos, que empreguem processos construtivos de alvenaria estrutural e tem como uma de suas exigências o valor mínimo de resistência característica $f_{b k}=3,0 \mathrm{MPa}$ para o bloco estrutural empregado na construção. 
- Blocos queimados a $700^{\circ} \mathrm{C}$ tiveram média de 2,89 MPa tanto pelo Método Gráfico de Probabilidade, quanto pelo Método da Máxima Verossimilhança. A diferença de aproximadamente $2 \%$ no Módulo de Weibul $(\beta)$ entre os dois métodos não é significativa. O Método Gráfico de Probabilidade apresenta um $\mathrm{R}^{2}$ de 0,9019 , um desvio padrão de $0,7932 \mathrm{MPa}$ e uma tensão de ruptura característica $(\theta)$ de $3,2 \mathrm{MPa}$. Os limites inferior e superior encontrados pelo Método da Máxima Verossimilhança são 2,83 MPa e 3,56 MPa respectivamente.

- Blocos queimados a $800^{\circ} \mathrm{C}$ tiveram média de 3,93 MPa tanto pelo Método Gráfico de Probabilidade, quanto pelo Método da Máxima Verossimilhança. A diferença de aproximadamente $1 \%$ no Módulo de Weibul $(\beta)$ entre os dois métodos não é significativa. O Método Gráfico de Probabilidade apresenta um $\mathrm{R}^{2}$ de 0,9368 , um desvio padrão de $0,7345 \mathrm{MPa}$ e uma tensão de ruptura característica $(\theta)$ de 4,22 MPa. Os limites inferior e superior encontrados pelo Método da Máxima Verossimilhança são 3,91 MPa e 4,55 MPa respectivamente.

- O tratamento térmico em forno controlado a $800^{\circ} \mathrm{C}$ indica que os blocos podem entrar em um patamar de conformidade com a norma [8]. A resistência característica $f_{b k}=3,03$ $\mathrm{MPa}$ encontrada pela formulação da norma e o limite inferior encontrado pelo Método da Máxima Verossimilhança de 3,91 MPa guardam uma diferença de aproximadamente $29 \%$, porém ambos demonstram a conformidade dos blocos queimados a $800^{\circ} \mathrm{C}$.

- Os blocos queimados a $700^{\circ} \mathrm{C}$ apresentaram resistência característica $f_{b k}=1,64 \mathrm{MPa}$ pela formulação da norma [8] e o limite inferior encontrado pelo Método da Máxima Verossimilhança de 2,83 MPa, uma diferença de aproximadamente $72 \%$. No entanto, ambos os métodos indicam a não conformidade dos blocos queimados a $700^{\circ} \mathrm{C}$.

- Dada a grande dispersão na grandeza estudada faz-se necessário que seja empregado um número maior de elementos para análise por meio do Modelo de Frequência de Weibull. O número de 13 blocos recomendado pela norma [8] não é o suficiente para uma comparação segura com o Modelo de Frequência de Weibull. Os blocos queimados a $800^{\circ} \mathrm{C}$ alcançaram um indicativo mais aproximado do que os blocos queimados a $700^{\circ} \mathrm{C}$ demonstrando também a influência do tratamento térmico em suas propriedades.

\section{REFERÊNCIAS}

1 ALEXANDRE, J. Analysis of the raw materials and composition of a clay body used in red ceramics (in Portuguese). D.Sc. dissertation in Geotechnical Engineering Science. State University of the Northern Rio de Janeiro, UENF, Campos dos Goytacazes, RJ, Brazil. (2000), 174 pp.

2 Brazilian Association for Technical Norms - ABNT. NBR 7181 Determination of the particle size distribution of soils (in Portuguese), Rio de Janeiro, Brazil, corrected version, 1988

the plasticity limit in soils (in Portuguese). Rio de Janeiro, Brazil, corrected version, 1988.

through a $4.8 \mathrm{~mm}$ sieve - Determination of the density (in Portuguese). Rio de Janeiro, Brazil, 1984.

NBR 6.459 : 1984. Solo. Esta Norma prescreve o método para a determinação do limites de liquidez dos solos. Rio de Janeiro, RJ, 1984.

NBR 6.508 : 1984. Solo. Esta Norma prescreve o método de determinação da massa específica dos grãos de solos que passam na peneira de 4,8 $\mathrm{mm}$. Rio de Janeiro, RJ, 1984.

NBR 6457: 1986. Solo. Esta Norma prescreve o método para a preparação de amostras de solos para os ensaios de compactação e de caracterização. Rio de Janeiro, RJ, 1986. 
(in Portuguese). Rio de Janeiro, Brazil, 2005.

NBR 15.270 Ceramic Components

9 DE CARVALHO, E.A. Ensaios Mecânicos Parte II. Notas de aula. State University of the Northern Rio de Janeiro, UENF, Campos dos Goytacazes, RJ, Brazil. (2013).

10 DE CARVALHO, E.A. Avaliação da influência do fator de localização e rank mediano no valor do módulo de Weibull em materiais frágeis. In: 62ํㅡㄹ Congresso Anual da ABMVitória, ES, Brasil, 2007.

11 Instituto Brasileiro de Geografia e Estatística (IBGE) - CENSO 2010 Disponível em: $<$ http://www.ibge.gov.br/>.

12 MILLER, C. P. Determinação de parâmetros mecânicos e modelagem numérica em alvenaria estrutural de blocos cerâmicos da indústria de Campos dos Goytacazes. Dissertação (Mestrado) Engenharia Civil - Universidade Estadual do Norte Fluminense, UENF. Campos dos Goytacazes- RJ, 2008. 161p.

13 SABBATINI, F. H. Alvenaria estrutural: materiais, execução da estrutura e controle tecnológico. Caixa Econômica Federal, 2003.36 p.

14 DODSON, B. The Weibull Analysis Handbook, 2nd ed., ASQ Quality Press, 2006.

15 PEDROTI, L.G. Estudo de conformidades em relação à ABNT de blocos cerâmicos prensados e queimados. Dissertação (Mestrado) Engenharia Civil -Universidade Estadual do Norte Fluminense , UENF. Campos dos Goytacazes - RJ ,2007.97 p.

16 XAVIER, G.C. Resistência, alterabilidade e durabilidade de peças cerâmicas vermelhas incorporadas com resíduo de granito. Tese ( Doutorado) Engenharia Civil. Universidade Estadual do Norte Fluminense, UENF- Ciências de Engenharia - Campos dos Goytacazes- RJ, 2006. 202 p. 